\title{
Why Open Access?
}

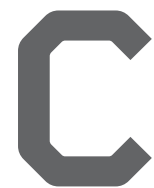

ONCERNING THE PROPOSED Research Works Act, introduced in the U.S. Congress in 2011, Moshe Y. Vardi's "Editor's Letter" "Why ACM?" (Sept. 2012) left me a little confused as to Vardi's own position on both the law and open access. He wrote, “...most people who tell me that ACM should adopt open access seem to equate open access with free access, as in "no one pays." Open access implies free access, but free access does not automatically imply some kind of free lunch or lead to a situation where no one pays. Taxpayers have already paid for the research and its access by funding the research.

Interpreting Vardi, the ACM position would seem to be: No implicit support for open access despite implicit support for the RWA by taking a neutral position. A respected professional scientific association like ACM should not take a neutral position here, as suggested by (past) ACM president Alain Chesnais in his President's blog earlier this year (http:// blog.acm.org/president/?p=67).

At least two well-respected professional associations - the Optical Society of America (OSA) and the Institute of Physics (IOP)-do support open access, integrating the practice into their publishing policies and operations. Ignoring such support for open access would represent gross arrogance on the part of ACM; see the OSA and IOP positions spelled out at http://www.opticsinfobase.org/submit/review/pub_charge.cfm\#OA and http://iopscience.iop.org/info/page/ openaccess. OSA also supports the idea of sponsorship; for example, any existing article can be made open access to the general public regardless of its publication date by subsidizing the cost of publication, usually including for peer reviews, lifetime operation, and long-term maintenance of the article in the organization's digital library. So Vardi's argument that open access means a financial burden for ACM does not hold up entirely.
Dissemination of knowledge is a core ACM value involving access to knowledge and pursuit of scientific inquiry. ACM must weigh its position as a not-for-profit professional scientific association, not as a commercial publisher. I appreciate that publishing is costly, but in almost all cases I know the cost of publishing is covered through government research grants.

Open access to publicly funded scientific research in the public interest is fundamental to an open society and to ACM's own values. ACM should therefore move toward some kind of open-access publishing. Perhaps it can introduce optional open access under certain conditions following the OSA and IOP models.

Mehmet Süzen, Bonn, Germany

I was impressed by Moshe Y. Vardi's willingness to engage the ACM membership, including me (Lifetime Member since 2008), concerning open-access publishing (Sept. 2012), and by ACM for not being dogmatic about its copyright policy (such as by not requiring copyright transfer in all instances, like IEEE, and by introducing Author-Izer). I was also pleasantly surprised to see that publishing is the source of only one-third of ACM's annual revenue stream, which represents a great opportunity to develop a business model that would enable (much more free) access to (many more) people. ACM could easily get stuck in its current publishing model, blinding itself to potentially groundbreaking opportunities; for a sad example of such ostrich-like thinking, see the recording industry.

As a way to help ACM move in the right direction, please consider the following:

opt-in. Provide an opt-in option for, say, a higher-price level of membership. In particular, some members whose subscription fee is paid by universities and employers or who are able to claim that fee on their tax returns might be willing to take such an option. Some current members might be willing to pay more simply because they see the goal (and the organization) as worthwhile and worthy, the way some people donate to keep Wikipedia free;

Crowd-funding. Determine the amount ACM needs to publish all its articles and papers in a year, then ask ACM members, libraries, foundations, and other subscribers to contribute toward that cost-basically a form of crowd-funding. (Only if the amount is actually raised would ACM then make the articles and papers available.); and

Set free. Determine the amount needed to make a paywalled article available for free, amending it to each article and paper. If potential readers would be willing to pay that amount or make a micro-donation (such as via Flatter), then the amount would go toward the goal until the article or paper becomes freely available to all.

ACM could develop an innovative business model leading to totally free access, not as in "no one pays" but as in redistributing who pays.

Holger Kienle, Berlin, Germany

\section{Author's Response:}

Süzen seems to have misread my editorial. Please note ACM issued a clear statement against RWA: ACM supports open access in several ways, including allowing personal and institutional repositories and the Author-Izer service. Dissemination of knowledge is a key value of ACM, but as ACM members, we need to decide how to cover the cost of publishing; for example, both OSA and IOP allow authors to pay to open access to their articles. ACM is considering adopting this approach.

Moshe Y. Vardi, Editor-in-Chief

\section{Not the Database World We Know} Communications readers have a right to expect accuracy. Sadly, accuracy is not always what they get. The article "All Your Database Are Belong to Us" by Erik Meijer (Sept. 2012) contains so many inaccuracies, confusions, and errors regarding "the database 
world" it is difficult to read coherently. The first paragraphs alone contain more egregious misstatements than most entire articles or papers. For the record: "The raw physical data model" is categorically not "at the center of the [relational database] universe." Queries do not "assume intimate details of the data representation (indexes, statistics, metadata)." While database technology relies on "The Closed World Assumption," this assumption has nothing to do with what the author apparently meant. Every phrase in "Exposing naked data and relying on declarative magic becomes a liability" relies on at least one counterfactual. "Objects should hide their private data representation, exposing it only via well-defined behavioral interfaces." But this is exactly what the relational model does-except (unlike OO) it adopts an interface discipline that makes ad hoc query and the like possible. "In the realm of [data] modelers, there is no notion of data abstraction." Astoundingly wrong. "[Database technology necessarily involves] a computational model with a limited set of operations." False. Although the (very powerful, well-defined, provably correct) required set of relational operations is small, the sky's the limit on derived relational operations or operations that define abstract data type/domain behavior.

The author's unfounded antipathy toward relational databases dominates even his application of CAP: "The problem with SQL databases... is the assumption that the data... meets a bunch of consistency constraints that is difficult to maintain in an open ['anything goes'?] distributed world." CAP does not eliminate this requirement; "...the hidden cost of forfeiting [system-enforced] consistency... is the need [for the programmer] to know the system's invariants." ${ }^{1}$ Nor can programmers "...design their systems to be robust...to inconsistency." Once data inconsistency invades a computationally complete system, it is not even, in general, detectable, and all bets are off. Consistency must be enforced, hence constraints. The author seemed to equate detecting abnormal execution with enforcing logical data consistency. No wonder confusion abounds; CAP consistency is single-copy consistency, a subset of what ACID databases provide, yet the Gilbert/Lynch CAP proof relies on linearizability, a more stringent requirement than the serializability ACID databases need or use.

And so on... Deconstructing the entire article properly would take more time than we care to devote, but the foregoing should suffice to demonstrate its fallaciousness. We hope the author is not teaching these confusions, errors, logical inconsistencies, and fallacies.

It is difficult even to believe the article was peer reviewed. Indeed, it is truly distressing it did not demonstrate even minimal understanding of one of the most important contributions to computing: the relational model. We can only deplore Communications's role in promulgating such a lack of understanding.

C.J. Date, Healdsburg, CA,

and D. McGoveran, Boulder Creek, CA

Reference

1. Brewer, E. CAP twelve years later: How the 'rules' have changed. IEEE Computer 45, 2 (Feb. 2012), 23-29.

\section{Author's Response:}

The purpose of the article was not to criticize the relational model but to point out how building industrial-strength systems using today's relational database systems requires leaving the ivory tower and dealing with a morass of ad hoc extensions to the clean mathematical basis of first-order predicate logic. Rather than depend on pure sets and relations, developers need to think in terms of (un)ordered multisets. For the sake of efficiency and lock-contention avoidance, transactions allow for various isolation levels that clearly violate the ACID guarantees of Platonic transactions. The article also considered whether in the new world of the Cloud we should view as complementary computational models that fundamentally address loosely coupled distributed systems, like Carl Hewitt's Actors.

Erik Meijer, Delft, The Netherlands

Communications welcomes your opinion. To submit a Letter to the Editor, please limit yourself to 500 words or less, and send to letters@cacm.acm.org.

(C) 2012 ACM 0001-0782/12/12

\section{Human Mobility}

Characterization from

Cellular Network Data

\section{Abstractions \\ for Genomics}

Computer Security

and the Modern Home

What College

Could Be Like

Conference-Journal

Hybrids

Browser Security:

Appearances

Can Be Deceiving

The Web Won't

Be Safe or Secure

Until We Break It

Condos

and Clouds

How Mechanical

Assemblies Work

Plus the latest news about side-channel attacks, beyond Hadoop, and stealing others' content. 